\title{
Defined Contingent On Relationship Pause Quantity
}

National Cancer Institute

\section{Source}

National Cancer Institute. Defined Contingent On Relationship Pause Quantity. NCI

Thesaurus. Code C93763.

A quantity of time that should elapse between when an activity is ready for execution and the actual beginning of the execution. 